\title{
Mutations in C-natriuretic peptide (NPPC): a novel cause of autosomal dominant short stature
}

\author{
Alfonso Hisado-Oliva, PhD ${ }^{1,2,3}$, Alba Ruzafa-Martin, MSc ${ }^{1}$, Lucia Sentchordi, MD, MSc ${ }^{1,3,4}$, \\ Mariana F.A. Funari, MSc ${ }^{5}$, Carolina Bezanilla-López, MD ${ }^{6}$, Marta Alonso-Bernáldez, MSc ${ }^{1}$, \\ Jimena Barraza-García, MD, MSc ${ }^{1,2,3}$, Maria Rodriguez-Zabala, MSc ${ }^{1}$, Antonio M. Lerario, MD, $\mathrm{PhD}^{7,8}$, \\ Sara Benito-Sanz, PhD ${ }^{1,2,3}$, Miriam Aza-Carmona, PhD ${ }^{1,2,3}$, Angel Campos-Barros, PhD ${ }^{1,2}$, \\ Alexander A.L. Jorge, MD, $\mathrm{PhD}^{5,7}$ and Karen E. Heath, $\mathrm{PhD}^{1,2,3}$
}

\begin{abstract}
Purpose: C-type natriuretic peptide (CNP) and its principal receptor, natriuretic peptide receptor B (NPR-B), have been shown to be important in skeletal development. CNP and NPR-B are encoded by natriuretic peptide precursor-C $(N P P C)$ and natriuretic peptide receptor 2 (NPR2) genes, respectively. While NPR2 mutations have been described in patients with skeletal dysplasias and idiopathic short stature (ISS), and several Npr2 and Nppc skeletal dysplasia mouse models exist, no mutations in NPPC have been described in patients to date.
\end{abstract}

Methods: NPPC was screened in 668 patients (357 with disproportionate short stature and 311 with autosomal dominant ISS) and 29 additional ISS families in an ongoing whole-exome sequencing study.

Results: Two heterozygous NPPC mutations, located in the highly conserved CNP ring, were identified. Both showed significant reductions in cyclic guanosine monophosphate synthesis, confirming their pathogenicity. Interestingly, one has been previously linked to skeletal abnormalities in the spontaneous $N p p c$ mouse long-bone abnormality (lbab) mutant.

Conclusions: Our results demonstrate, for the first time, that NPPC mutations cause autosomal dominant short stature in humans. The NPPC mutations cosegregated with a short stature and small hands phenotype. A CNP analog, which is currently in clinical trials for the treatment of achondroplasia, seems a promising therapeutic approach, since it directly replaces the defective protein.

Genet Med advance online publication 29 June 2017

Key Words: CNP; FGFR3; NPPC; NPR2; short stature

\section{INTRODUCTION}

C-type natriuretic peptide (CNP) and its principal receptor, natriuretic peptide receptor B (NPR-B), have been shown to be important in cartilage homeostasis and endochondral bone formation. ${ }^{1-4}$ Binding of CNP to the NPR-B receptor induces cyclic guanosine monophosphate (cGMP) synthesis, activating an intracellular signaling cascade that mediates its effects. ${ }^{5,6}$ In humans, CNP and NPR-B are encoded by the natriuretic peptide precursor C (NPPC; MIM 600292) and natriuretic peptide receptor 2 (NPR2; MIM 108961) genes, respectively. Homozygous defects in NPR2 cause a severe skeletal dysplasia, acromesomelic dysplasia Maroteaux type (MIM 602875), which is characterized by severe short stature ( $<5$ standard deviations from the mean (SDS) in adults), phalangeal-metacarpal abnormalities, and shortening and bowing of the limbs. ${ }^{4}$ Interestingly, heterozygous NPR2 defects have been described in some idiopathic short stature (ISS; MIM 616255) cases, and in patients presenting with some clinical features similar to Léri-Weill dyschondrosteosis (LWD; MIM 127300), such as shortening of the limbs and metacarpals, bowing of the radius, cleft palate and muscular hypertrophy, but lacking the characteristic Madelung deformity typically associated with LWD. ${ }^{7-10}$

In contrast, no NPPC mutations have been observed in humans to date, although the importance of $N p p c$ for skeletal growth has been clearly demonstrated in mice. The Nppc knockout mouse is characterized by a severe disproportionate form of dwarfism, associated with shortening of the limbs and endochondral ossification defects resulting in postnatal death. ${ }^{1}$ Cnp overexpression leads to overgrowth in transgenic mice. ${ }^{11}$ In humans, several genome-wide association studies have established a relationship between NPPC and height. ${ }^{12-14}$ Furthermore, it has been hypothesized that CNP haploinsufficiency may be the cause of the short stature observed in an individual with a $1.9 \mathrm{Mb}$ deletion in chromosome $2 \mathrm{q} 37$, which spans 20 genes, including NPPC. ${ }^{15}$ Conversely, NPPC overexpression due to balanced translocations have been observed in individuals with tall stature and

\footnotetext{
${ }^{1}$ Institute of Medical and Molecular Genetics (INGEMM), Hospital Universitario La Paz, Universidad Autónoma de Madrid, IdiPAZ, Madrid, Spain; ${ }^{2}$ Centro de Investigación Biomédica en Red de Enfermedades Raras (CIBERER, U753), Instituto Carlos III, Madrid, Spain; ${ }^{3}$ Multidisciplinary Skeletal Dysplasia Unit (UMDE), Hospital Universitario La Paz, Madrid, Spain; ${ }^{4}$ Department of Pediatrics, Hospital Universitario Infanta Leonor, Madrid, Spain; ${ }^{5}$ Laboratorio de Hormonios e Genetica Molecular (LIM42), Hospital das Clinicas da Faculdade de Medicina, Universidade de São Paulo (USP), São Paulo, Brazil; ${ }^{6}$ Department of Pediatrics, Hospital Universitario Fundación Alcorcón, Madrid, Spain; ${ }^{7}$ Unidade de Endocrinologia Genetica (LIM25), Faculdade de Medicina da Universidade de São Paulo (USP), São Paulo, Brazil; ${ }^{8}$ Division of Metabolism, Endocrinology and Diabetes, Department of Internal Medicine, University of Michigan, Ann Arbor, Michigan, USA. Correspondence: Karen E. Heath (karen.heath@salud.madrid.org) 
long hands, feet, and halluces, together with other skeletal defects. ${ }^{16}$

Since similar under- or overgrowth phenotypes have been observed in mice with Nppc or Npr2 mutations, and NPR2 mutations have been associated with short stature and skeletal defects in humans, we hypothesized that NPPC mutations might be associated with similar phenotypes in humans.

Here we present, for the first time, the identification of heterozygous mutations in NPPC in two families with short stature and small hands. Both mutations are located within the highly conserved ring structure of CNP. They failed to activate NPR-B in the homozygous state, and showed a significant reduction in cGMP synthesis in the heterozygous state. Interestingly, one of the identified NPPC missense mutations, p.Arg117Gly, is identical to that described in the spontaneous Nppc long-bone abnormality (lbab) mouse mutant, with shortening of long bones and smaller growth plates. A CNP analog that directly replaces the defective protein is currently in clinical trials for the treatment of achondroplasia and seems a promising therapeutic approach for patients with NPPC mutations causing short stature.

\section{MATERIALS AND METHODS}

\section{Cohorts}

We undertook NPPC mutation screening in two cohorts of short stature patients. All participants provided informed consent for the performed studies and ethical approval was obtained from the respective institutions.

The first cohort consisted of 668 patients with disproportionate short stature $(n=357)$ or a diagnosis of ISS (i.e., proportionate short stature and no hormone deficiencies; $n=311$ ). SHOX and NPR2 alterations were previously excluded. A second cohort consisting of 29 families with ISS in which SHOX and NPR2 alterations had also been previously excluded was screened in the context of an ongoing whole-exome sequencing study.

\section{Family descriptions Family 1}

In family 1 , the proband was a 12-year-old Spanish girl (III.1), who was referred to her local endocrinology clinic for short stature (-2.3 SDS; Figure 1). Bone age was concordant for chronological age and her insulin-like growth factor 1 $(201 \mathrm{ng} / \mathrm{ml} ;-1.27$ SDS) and insulin-like growth factor binding protein 3 ( -0.72 SDS) levels were normal. She had short hands and feet, a short fourth metacarpal, and hypertelorism. Her mother had a height of $148.6 \mathrm{~cm}(-2.6$ SDS) with small hands, and her father also had short stature $(164.3 \mathrm{~cm} ;-2.0 \mathrm{SDS})$, but normal hand length. The younger sister, aged 11.5 years, had normal height $(147.0 \mathrm{~cm} ;-0.4$ SDS) and hand length. All relatives had normal sitting height: height ratios and armspan:height ratios, indicating that they all had proportionate stature. At 13.5 years of age, the proband still presents with proportionate short stature $(-2.3$ SDS $)$ and the same dysmorphic features described at presentation. Further clinical details and anthropometric measurements of the proband and family members are shown in Table 1, Figure 1 and Supplementary Figure S1 online.

\section{Family 2}

In family 2 , the proband was a Brazilian girl (II.1), now aged 21 , with short stature $(145.0 \mathrm{~cm} ;-2.9$ SDS) and small hands after undergoing normal puberty. She is the older sister of a Brazilian male (II.2) who was referred to an endocrinology clinic for proportional short stature and delayed puberty. He was diagnosed with constitutional delay of growth (height: $141.0 \mathrm{~cm} ;-2.77 \mathrm{SDS}$ ) and puberty, and treated with testosterone to induce puberty at the age of 14.6 years (50 $\mathrm{mg}$ of testosterone cypionate intramuscularly every 4 weeks for 3 months). His current height at the age of 17.2 years is $161.5 \mathrm{~cm}(-2.0 \mathrm{SDS})$ and he continues to grow. Their father has short stature $(158.8 \mathrm{~cm} ;-2.4$ SDS $)$ and small hands, while their mother has normal height $(157.5 \mathrm{~cm} ;-0.8$ SDS) and no other relevant clinical history. Further clinical details and anthropometric measurements of the proband and family members are provided in Table $\mathbf{1}$ and Supplementary Figure S1.

\section{Genetic analysis}

In cohort 1, the coding sequences and intron:exon boundaries of NPPC (NM_024409.3) were analyzed by Sanger sequencing (oligonucleotides and PCR conditions are available on request). Conservation and pathogenicity prediction analysis of the identified NPPC variants was carried out using combined annotation-dependent depletion (CADD) version 1.3 (http://cadd.gs.washington.edu/) and Alamut version 2.8 software (Interactive Biosoftware, France), which included sorting intolerant from tolerant (SIFT; http://sift.bii.a-star. edu.sg), Polyphen (http://genetics.bwh.harvard.edu/pph2), MutationTaster (http://www.mutationtaster.org) and splicing predictor analyses. Population frequencies of the detected variants were assessed using the Exome Aggregation Consortium (ExAC) browser (http://exac.broadinstitute.org), 1000 Genomes data (http://www.internationalgenome.org) and 264 Spanish normal-height controls (Banco de ADN, Salamanca University).

In cohort 2, exome sequencing (SureSelect Target Enrichment System, Agilent Technologies, Santa Clara, CA) was undertaken according to standard protocols and sequences were generated on an Illumina HiSEQ 2500 platform running on paired-end mode. Reads were aligned to the hg19 assembly of the human genome with the BWA-MEM aligner. Duplicated reads were flagged with biobambam2. Variant calling was performed with freebayes and the resulting variant call formats were annotated with ANNOVAR (http://annovar. openbioinformatics.org). Nonsynonymous or splice-site variants were then selected based on the pedigree and the familial short stature. Subsequently, our variant filtering focused on variants absent in controls (ExAC, 1000 Genomes and our in-house databases), highly conserved (GERP++; http://mendel.stanford.edu/SidowLab/downloads/gerp) and 


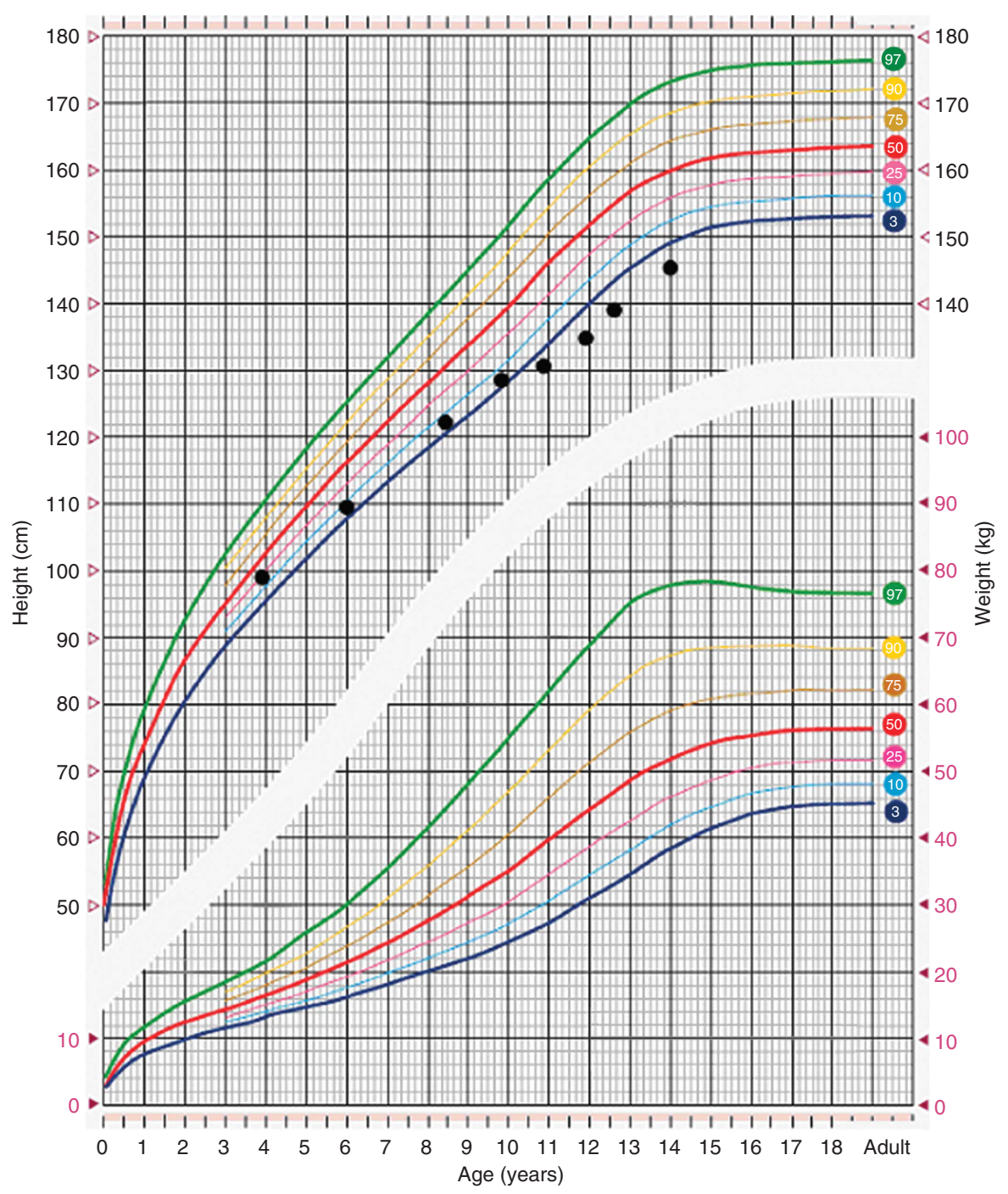

Figure 1 Growth curve of the female proband from family 1. The growth curve is based on 2010 Spanish height data (http://www.aeped.es/ noticias/estudios-espanoles-crecimiento-2010). The colored lines represent the growth percentiles.

predicted to be pathogenic by multiple in silico programs: Polyphen2, Mutation Assessor (http://mutationassessor.org/ r3), SIFT, PROVEAN (http://provean.jcvi.org/index.php) and CADD version 1.3 (Supplementary Figure S1). Variants were validated by Sanger sequencing.

\section{Plasmid constructions}

The Myc-tagged expression constructs containing the detected NPPC mutants were generated by site-directed mutagenesis according to the manufacturer (QuikChange Site-Directed Mutagenesis Kit; GE Healthcare, Uppsala, Sweden) of pMyc-CNP (NM_024409; Origene Technologies, Rockville, MD). Mutagenesis primers (mutated base underlined) for the p.Arg117Gly (R117G) mutation were 5'-GGC CTCAAGCTGGACGGAATCGGCTCCATGAGC-3', and for the p.Gly119Cys (G119C) mutation they were 5'-GGCCT CAAGCTGGACCGAATCTGCTCCATGAGC-3'. All constructs were verified by sequencing.

\section{cGMP synthesis}

COS-7 cells were transfected with $500 \mathrm{ng}$ of the complementary DNA of NPR2 wild type (pHA - NPR-B) or empty vector (pHA) and $500 \mathrm{ng}$ of the complementary DNA of NPPC wild type, one of the two mutations, or a combination of the wild type and one of the mutations ( $250 \mathrm{ng}+250 \mathrm{ng}$ ), thus mimicking the heterozygous state, using a 3:2 FuGene 6 (Promega, Madison, WI):DNA ratio according to the manufacturer's instructions. After $48 \mathrm{~h}$ posttransfection, $0.1 \mathrm{M}$ hydrogen chloride was added to stop endogenous phosphodiesterase activity, to stabilize the released cGMP, and to lyse the cells. Cell lysates were centrifuged at $600 \mathrm{~g}$ for 10 minutes and the supernatants were isolated.

The cGMP levels in the supernatants were measured using the cGMP Complete ELISA kit (ENZO Life Sciences, Farmingdale, NY). Each construct combination was transfected in triplicate, each transfection was assayed in triplicate, and three biological replicates were performed. Student's $t$-tests, using SPSS version 15.0 for Windows, were carried out 


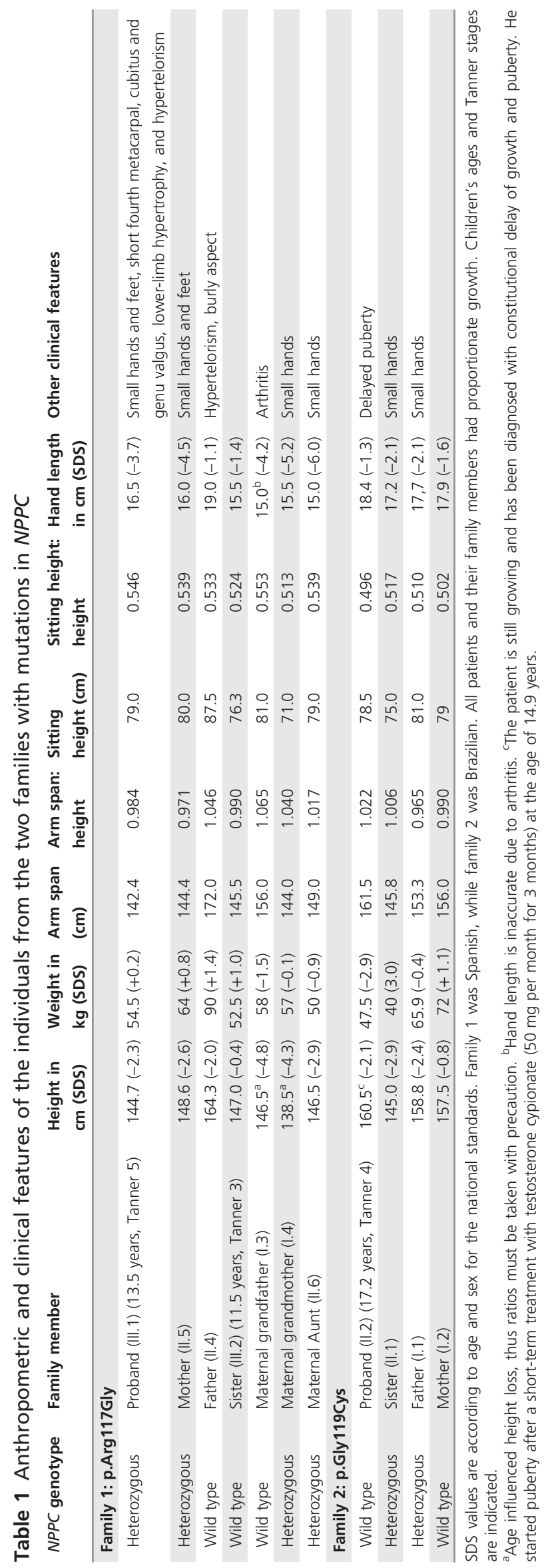

to compare the differences between wild type and mutants. A $P$ value of $<0.05$ was considered statistically significant.

\section{RESULTS}

Two heterozygous NPPC variants, p.Arg117Gly and p. Gly119Cys, were detected in two unrelated short stature individuals (Figure 2 and Table 2). The NPPC variant, p. Arg117Gly (family 1) was detected in the autosomal dominant ISS cohort. It affects an amino acid within the ring structure of CNP, which is highly conserved between the three natriuretic peptides, CNP, brain natriuretic peptide (BNP), and atrial natriuretic peptide (ANP; Figure 2). ${ }^{17}$ In silico analysis predicts the variant to be deleterious (Table 2). The variant is absent from the large population databases (Exome Aggregation Consortium and 1000 Genomes) and from 264 Spanish individuals with normal stature $(-2$ to +2 SDS) from the DNA bank at the University of Salamanca (http://www.bancoadn.org/). No further mutations were identified after additional screening of this patient using a targeted next-generation sequencing skeletal dysplasia panel (SKELETALSEQ.V4; $n=327$ genes).

The heterozygous NPPC variant c.349C > G (p.Arg117Gly) was identified in the proband of family 1 (III.1), who had short stature (-2.3 SDS), small hands and feet, shortening of the fourth metacarpals, and hypertelorism. Subsequent analysis confirmed the presence of the NPPC mutation in the similarly affected mother, maternal grandmother and aunt, while it was absent in the examined family members with normal stature (Figure $\mathbf{2 a}$ and Table $\mathbf{1}$ ).

The second heterozygous NPPC variant, c.355G > T (p.Gly119Cys), was detected by WES in a Brazilian adult female (II.1) and her father (family 2), both of whom presented with short stature and small hands (Figure $\mathbf{2 b}$ and Table 2). The variant was absent from the normal stature mother and the short stature brother (II.2), who was diagnosed with constitutional growth and pubertal delay and who has a predicted adult height within the normal range (Supplementary Figure S3). Both the mother and the brother had normal hand size. The p.Gly119Cys variant also affects a highly conserved amino acid located in the CNP ring, two amino acids adjacent to the mutation present in family 1 (Figure 2c). This amino acid is conserved between CNP and ANP, but not BNP. The c.355G > T variant is absent from the large population databases and in silico analyses predict the variant to be pathogenic (Table 2). No additional pathogenic variants were identified by WES to explain the short stature in the proband or her family (Supplementary Figure S2, Tables S1 and S2).

In order to functionally confirm the predicted pathogenicity of the identified NPPC variants, we analyzed their ability to activate the NPR-B receptor by assessing the CNP-dependent guanylate cyclase. Both NPPC variants, p.Arg117Gly and p. Gly119Cys, failed to activate NPR-B in the homozygous state and showed a significant reduction in CGMP synthesis in the heterozygous state when cotransfected (ratio 1:1) with the wild-type construct (Figure 3). 
a

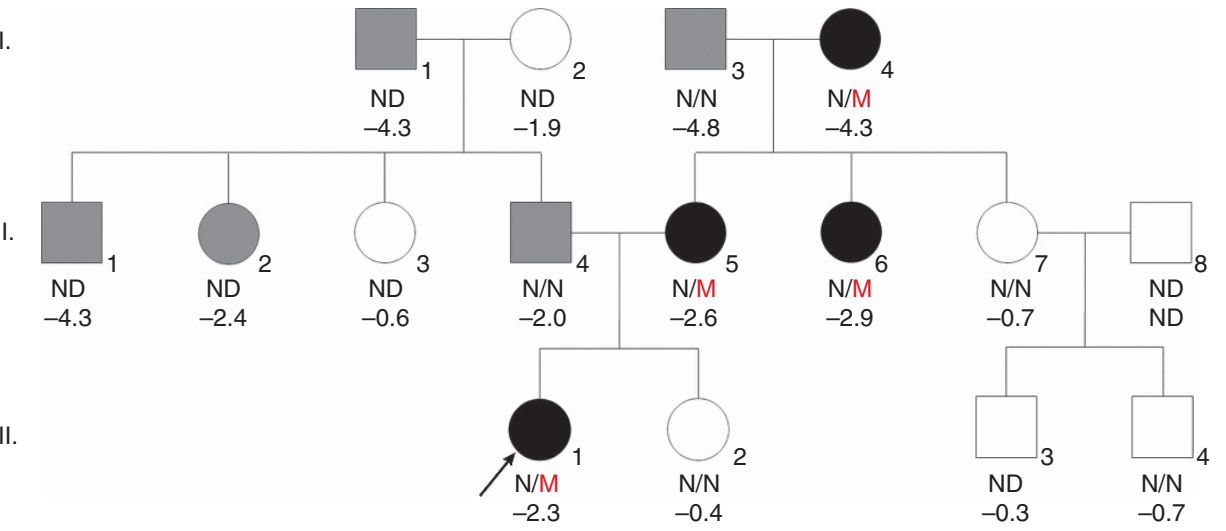

b
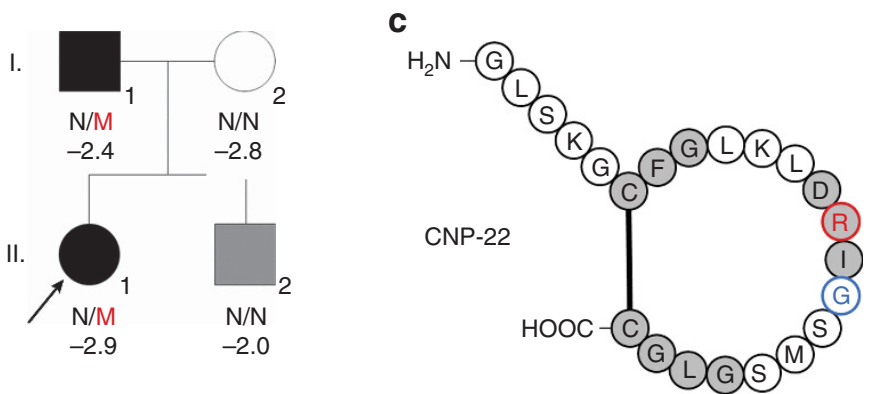

Figure 2 Pedigrees and cosegregation analysis of the two families carrying the NPPC variants. Cosegregation analysis of the heterozygous NPPC variants, c.349C > G (p.Arg117Gly) from family 1 (a) and c.355G > T (p.Gly119Cys) from family 2 (b). NPPC genotype (N: wild type and M: mutated allele) and height SDS are indicated below each individual. The height SDS for individual II.2 (b) is currently on rhGH treatment. Individuals with short stature and small hands or short stature only are indicated with black and gray symbols, respectively. Probands are indicated by arrows. (c) Schematic representation of the CNP-22 functional ring showing the localization of the two mutated amino acids, p.Arg117Gly and p.Gly119Cys, in red and blue, respectively. Arg117 is conserved in all three natriuretic peptides (ANP, BNP and CNP), while Gly119 is conserved in CNP and ANP.

\section{DISCUSSION}

Our results demonstrate, for the first time, that NPPC mutations cause autosomal dominant short stature in humans. In both families, the NPPC mutation cosegregated with the short stature and small hands phenotype, and was absent in unaffected individuals, thus indicating complete penetrance. The profound negative effect on cGMP generation shown by the functional assessment of both mutations further corroborates these observations.

Interestingly, one of the identified NPPC missense mutations, p.Arg117Gly, is identical to that described in the spontaneous Nppc long-bone abnormality (lbab) mouse mutant. ${ }^{18}$ The lbab/lbab mouse is characterized by dwarfism due to the shortening of long bones and growth plate narrowing, especially in the proliferative and hypertrophic zones. ${ }^{19,20}$ Although the heterozygous mice $(+/ / b a b)$ appeared to show no remarkable phenotype, a closer examination of the growth plate revealed a slight reduction in $\operatorname{size}^{20}{ }^{20}$ thus correlating with the mild phenotype observed in humans.

NPPC encodes the 126-amino acid pre-pro CNP. The 23residue signal peptide is proteolytically cleaved, giving rise to the pro-CNP peptide, which is further processed by the prohormone convertase furin into two secreted peptides: the propeptide $1-50$ and CNP-53. ${ }^{21}$ In some tissues, CNP-53 is then subsequently cleaved by an unknown extracellular enzyme to generate CNP-22. These mature peptides contain a conserved 17-amino acid disulfide-linked ring, necessary for correct functioning. Although CNP-22 and CNP-53 have very similar NPR-B activation and binding profiles, their tissue expression differs. While CNP-53 is the predominant form in the brain, endothelial cells, and heart, CNP-22 is higher in the plasma and cerebral spinal fluid. ${ }^{21}$

Both mutations are located within the ring structure of $\mathrm{CNP}$, which is highly conserved between the three natriuretic peptides CNP, BNP, and ANP. Molecular modeling of the p.Arg117Gly mutation suggested that the presence of a glycine at codon 117 provides CNP with higher conformational flexibility, thus impeding the interaction between CNP and its receptor, NPR-B. ${ }^{22}$

The prevalence of NPPC mutations in the entire cohort of short stature individuals $(n=340)$ was $\sim 0.6 \%$. The prevalence of NPPC mutations might be lower in an unselected cohort of ISS children, thus the screening of a large cohort is required to determine a more accurate estimate of the prevalence. Moreover, a previous report indicated that NPPC large deletions may be associated with short stature, ${ }^{15}$ thus suggesting that the calculated prevalence might be underestimated since deletions were not screened in the current 
Table 2 Genetic details and in silico analysis of the NPPC variants

\begin{tabular}{|c|c|c|c|c|c|c|c|c|c|c|c|}
\hline Pt no. & CDNA & Exon & Amino acid & Domain & Country & Cosegregation $^{a}$ & GERP++ & SIFT & Mutation taster & Polyphen & CADD v1.3 \\
\hline \multirow[t]{2}{*}{1} & c. $349 C>G$ & 2 & p.Arg117Gly & Ring & Spain & Yes & 4.4 & Del & Disease causing & Damaging & Del \\
\hline & & & & & & & & (0) & (1) & $(1.000)$ & (31) \\
\hline 2 & c. $355 \mathrm{G}>\mathrm{T}$ & 2 & p.Gly119Cys & Ring & Brazil & Yes & 5.29 & Del & Disease causing & Damaging & Del \\
\hline
\end{tabular}

CDNA, complementary DNA; Del, deleterious.

All variants were absent from the ExAc database, 1000 Genomes and 264 normal-height Spanish individuals (DNA bank, University of Salamanca).

GERP++ values $>2.45$ are deemed conserved. CADD version 1.3 values $>20$ are classified as pathogenic. The probability of pathogenicity for Polyphen and MutationTaster are on a scale of $0-1$, with 1 indicating deleterious. In contrast, SIFT values are indicated on a scale of $0-1$ with 0 being deleterious and 1 indicating a polymorphism.

aCosegregation details are shown in Figure 2.

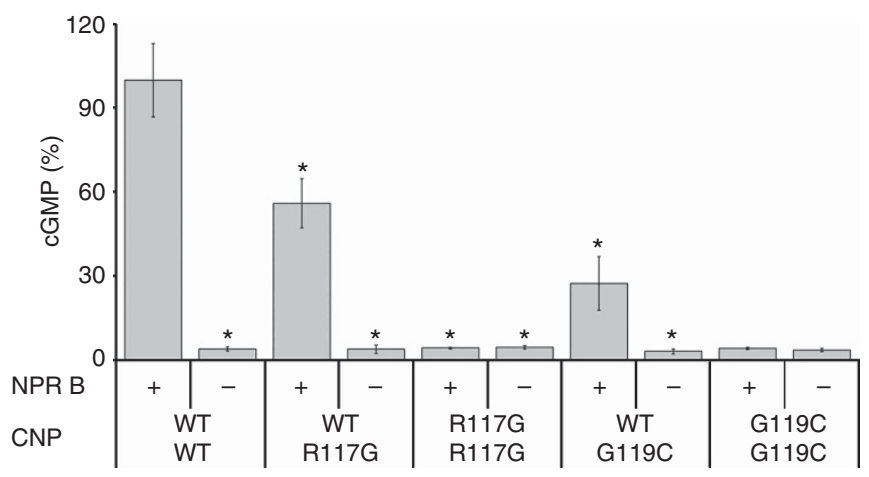

Figure 3 Functional characterization of the CNP variants. Analysis of the capacity of CNP wild type or the two mutants to activate NPR-Bmediated CGMP synthesis. CGMP activity of COS-7 cells transfected with NPR-B wild type $(+)$ or empty $(-)$, together with CNP wild type (WT) or mutants p.Arg117Gly (R117G) or p.Gly119Cys (G119C) and a 1:1 ratio of CNP wild type and each mutant was measured. CGMP values are expressed as a percentage relative to CNP/NPR-B-stimulated WT +/- SD. Each construct combination was transfected in triplicate, and each transfection was assayed in triplicate. Three biological replicates were performed. Thus, the statistics was determined for 27 ELISA assay results per construct combination. ${ }^{\star} P<0.01$. Both mutants showed a drastic reduction in CNP-dependent CGMP synthesis in the heterozygous state and total ablation in the homozygous state.

study. The estimated incidence of NPR2 mutations is five times higher, with mutations being detected in $\sim 3 \%$ of children initially classified as ISS and $\sim 3 \%$ of disproportionate short stature cases. ${ }^{8,9}$ Indeed, NPPC is considerably smaller than NPR2, thus the probability of a random mutation is far less, but this difference in incidence may also be due to other factors such as mutational hotspots or reproductive fitness.

Patients with heterozygous mutations in the gene encoding the ligand $(N P P C)$ or in the gene encoding its receptor (NPR2) share certain clinical features, such as short stature, small hands, or brachydactyly. Some individuals also present with cubitus valgus and a high-arched palate. However, individuals with NPR2 mutations may show additional clinical features resembling those observed in acromesomelic dysplasia Maroteaux type individuals, including frontal bossing and mesomelic shortening of the forearm. Although clinical heterogeneity is indeed observed, the principal difference appears to be that individuals with NPR2 mutations present with disproportionate growth while NPPC mutations to date have been observed only in individuals with proportionate growth. However, further cases are required for a more precise phenotypic evaluation.

A novel therapeutic approach for patients with NPPC mutations may be available in the near future. Phase II clinical trials (ClinicalTrials.gov identifier: NCT02055157) are currently in progress to test the efficacy of a CNP analog (BMN111, Biomarin Pharmaceutical) in patients with achondroplasia (MIM 100800), the most common type of dwarfism in humans. BMN111 acts as a key regulator of longitudinal bone growth by downregulating the mitogen-activated protein kinase pathway, which is activated by the FGFR3 (MIM 134934) gain-of-function mutation, p.Gly380Arg, present in 99.9\% of individuals with achondroplasia. This results in the restoration of chondrocyte proliferation and differentiation, leading to bone growth. Preliminary data show promising improvements in height and other clinical characteristics associated with this pathology, as previously observed in the preclinical studies, where the CNP analog rescued the skeletal phenotype in Fgfr3 Y367C/+ mice..$^{23,24}$ In the context of an NPPC mutation, the CNP analog seems a promising therapeutic approach, since it directly replaces the defective protein.

In summary, we have identified the first NPPC mutations, in patients with proportionate short stature and small hands. The incidence of NPPC mutations in our short stature cohort was $0.6 \%$. We also wait with great interest for the results of the CNP analog clinical trial as this would be a direct therapeutic approach for patients with CNP mutations.

\section{SUPPLEMENTARY MATERIAL}

Supplementary material is linked to the online version of the paper at http://www.nature.com/gim

\section{ACKNOWLEDGMENTS}

The authors thank the families for their participation in this study. This work was supported in part by the following grants: SAF2012-30871 and SAF2015-66831-R from the MINECO (to K.E.H), ENDOSCREEN (S2010/BMD-2396) from the Comunidad de Madrid (to K.E.H and A.C-B), 2013/03236-5 and 2014/50137 from the São Paulo Research Foundation (FAPESP), 
304678/2012-0 (to A.A.L.J.) from the National Council for Scientific and Technological Development (CNP), ISCIII (PI12100649) from the Fondo de Investigaciones Sanitarias (to A.C.-B), and an Eli Lilly research prize grant from the Spanish Pediatric Endocrinology society (Fundación SEEP) to K.E.H. A.H.-O. was the recipient of an FPI PhD studentship from the Basque Country, $\mathrm{M}$. R.-Z. received a fellowship from the Fundación SEEP, J.B.-G. received a CIBERER Lanzadera fellowship, and S.B.-S. received a postdoctoral CIBERER fellowship.

\section{DISCLOSURE}

The authors declare no conflict of interest.

\section{REFERENCES}

1. Chusho H, Tamura N, Ogawa $Y$, et al. Dwarfism and early death in mice lacking C-type natriuretic peptide. Proc Natl Acad Sci USA 2001;98: 4016-4021.

2. Tamura N, Doolittle LK, Hammer RE, Shelton JM, Richardson JA, Garbers DL Critical roles of the guanylyl cyclase $B$ receptor in endochondral ossification and development of female reproductive organs. Proc Natl Acad Sci USA 2004;101:17300-17305.

3. Tsuji T, Kunieda T A loss-of-function mutation in natriuretic peptide receptor 2 (Npr2) gene is responsible for disproportionate dwarfism in cn/ cn mouse. J Biol Chem 2005;280:14288-14292.

4. Bartels CF, Bukulmez $H$, Padayatti $P$, et al. Mutations in the transmembrane natriuretic peptide receptor NPR-B impair skeletal growth and cause acromesomelic dysplasia, type maroteaux. Am J Hum Genet 2004;75:27-34.

5. Ozasa A, Komatsu Y, Yasoda A, et al. Complementary antagonistic actions between C-type natriuretic peptide and the MAPK pathway through FGFR-3 in ATDC5 cells. Bone 2005;36:1056-1064.

6. Krejci $P$, Masri $B$, Fontaine $V$, et al. Interaction of fibroblast growth factor and $\mathrm{C}$-natriuretic peptide signaling in regulation of chondrocyte proliferation and extracellular matrix homeostasis. J Cell Sci 2005;118: 5089-5100.

7. Olney RC, Bukulmez $\mathrm{H}$, Bartels CF, et al. Heterozygous mutations in natriuretic peptide receptor-B (NPR2) are associated with short stature. J Clin Endocrinol Metab 2006:91:1229-1232.

8. Vasques GA, Amano N, Docko AJ, et al. Heterozygous mutations in natriuretic peptide receptor-B (NPR2) gene as a cause of short stature in patients initially classified as idiopathic short stature. J Clin Endocrinol Metab 2013;98:E1636-E1644.

9. Hisado-Oliva A, Garre-Vázquez Al, Santaolalla-Caballero F, et al. Heterozygous NPR2 mutations cause disproportionate short stature, similar to Léri-Weill dyschondrosteosis. J Clin Endocrinol Metab 2015;100:E1133-E1142.

10. Wang SR, Jacobsen CM, Carmichael H, et al. Heterozygous mutations in natiuretic peptide receptor-B (NPR2) gene as a cause of short stature. Hum Mutat 2015;36:474-481.

11. Kake $\mathrm{T}$, Kitamura $\mathrm{H}$, Adachi $\mathrm{Y}$, et al. Chronically elevated plasma C-type natriuretic peptide level stimulates skeletal growth in transgenic mice. Am J Physiol Endocrinol Metab 2009;297:E1339-E1348.

12. Gudbjartsson DF, Walters GB, Thorleifsson G, et al. Many sequence variants affecting diversity of adult human height. Nat Genet 2008;40: 609-615.

13. Estrada K, Krawczak M, Schreiber S, et al. A genome-wide association study of northwestern Europeans involves the C-type natriuretic peptide signaling pathway in the etiology of human height variation. Hum $\mathrm{Mol}$ Genet 2009;18:3516-3524.

14. Wood AR, Esko T, Yang J, et al. Defining the role of common variation in the genomic and biological architecture of adult human height. Nat Genet 2014;46:1173-1186.

15. Tassano E, Buttgereit J, Bader M, et al. Genotype-phenotype correlation of 2 q37 deletions including NPPC gene associated with skeletal malformations. PLOS ONE 2013;8:e66048.

16. Moncla A, Missirian C, Cacciagli P, et al. A cluster of translocation breakpoints in 2 q37 is associated with overexpression of NPPC in patients with a similar overgrowth phenotype. Hum Mutat 2007;28:1183-1188.

17. Potter LR, Abbey-Hosch S, Dickey DM. Natriuretic peptides, their receptors, and cyclic guanosine monophosphate-dependent signaling functions. Endocr Rev 2006;27:47-72.

18. Jiao $\mathrm{Y}, \mathrm{Yan} J$, Jiao $\mathrm{F}$, et al. A single nucleotide mutation in $\mathrm{Nppc}$ is associated with a long bone abnormality in Ibab mice. BMC Genet 2007;8:16.

19. Tsuji T, Kondo E, Yasoda A, et al. Hypomorphic mutation in mouse Nppc gene causes retarded bone growth due to impaired endochondral ossification. Biochem Biophys Res Commun 2008;376:186-190.

20. Kondo E, Yasoda A, Tsuji T, et al. Skeletal analysis of the long bone abnormality (lbab/lbab) mouse, a novel chondrodysplastic C-type natriuretic peptide mutant. Calcif Tissue Int 2012;90:307-318.

21. Wu C, Wu F, Pan J, Morser J, Wu Q. Furin-mediated processing of pro-C-type natriuretic peptide. J Biol Chem 2003;278:25847-25852.

22. Yoder AR, Kruse AC, Earhart CA, Ohlendorf DH, Potter LR. Reduced ability of C-type natriuretic peptide (CNP) to activate natriuretic peptide receptor B (NPR-B) causes dwarfism in Ibab-/- mice. Peptides 2008;29: $1575-1581$

23. Lorget F, Kaci N, Peng J, et al. Evaluation of the therapeutic potential of a CNP analog in a Fgfr3 mouse model recapitulating achondroplasia. Am J Hum Genet 2012;91:1108-1114.

24. Wendt DJ, Dvorak-Ewell M, Bullens S, et al. Neutral endopeptidaseresistant $C$-type natriuretic peptide variant represents a new therapeutic approach for treatment of fibroblast growth factor receptor 3-related dwarfism. J Pharmacol Exp Ther 2015;353:132-149. 